\title{
Repercussões cardiorrespiratórias da COVID-19: uma revisão integrativa
}

\section{Cardiorespiratory repercussions of COVID-19: a integrative review}

Repercusiones cardiorrespiratorias de COVID-19: una revisión integrator

Antônio Miguel De Sales Filho ORCID: https://orcid.org/0000-0002-7247-039X Faculdade FACOTTUR, Brasil

E-mail: anttonio_miguel@hotmail.com

Roberta França de Aguiar ORCID: https://orcid.org/0000-0003-3137-6361 Faculdade FACOTTUR, Brasil

E-mail: BBbetafranca@gmail.com

Renata Alves Calixto da Silva ORCID: https://orcid.org/0000-0002-0119-2206 Faculdade FACOTTUR, Brasil

E-mail: renataalves248@gmail.com

Harrison Euller Vasconcelos Queiroz ORCID: https://orcid.org/0000-0001-7906-2981 Faculdade FACOTTUR, Brasil

E-mail: harrisoneuller@gmail.com

Joaci do Valle Nóbrega Júnior ORCID: https://orcid.org/0000-0002-8189-8865 Faculdade FACOTTUR, Brasil

E-mail: Joaci.vnjunior@gmail.com

Amanda Fonseca Do Nascimento ORCID: https://orcid.org/0000-0001-7804-671X Faculdade FACOTTUR, Brasil

E-mail: amandas2nascimento@gmail.com

José Rennan William Figueiredo Morais

ORCID: https://orcid.org/0000-0001-5204-3967 Faculdade FACOTTUR, Brasil

E-mail: rennan.william@hotmail.com

Mateus Porfirio Rodrigues

ORCID: https://orcid.org/0000-0002-6747-4898 Faculdade FACOTTUR, Brasil

E-mail: mateusporfirio6@gmail.com

Ingrid Larissa da Silva Laurindo

ORCID: https://orcid.org/0000-0001-7658-2455 Faculdade FACOTTUR, Brasil

E-mail: larissasilvalaurindo@gmail.com Vanessa Silva Lapa

ORCID: https://orcid.org/0000-0003-0944-7952 Faculdade FACOTTUR, Brasil

E-mail: Vanessa-lapa@hotmail.com

André Luís Silva dos Santos

ORCID: https://orcid.org/0000-0002-2865-6576 Faculdade FACOTTUR, Brasil

E-mail: andreluiis1@gmail.com

Klyvia Juliana Rocha de Moraes ORCID: https://orcid.org/0000-0001-7407-4027 Faculdade FACOTTUR, Brasil E-mail:klyviaj@yahoo.com.br 


\title{
Resumo
}

Objetivo: Difundir informações a respeito das repercussões cardiorrespiratórias da COVID-19. Metodologia: Revisão da literatura com levantamento bibliográfico nas bases de dados LILACS, SciELO, PubMed e PEDro considerando ensaios clínicos e estudos observacionais publicados no período de julho de 2020 à abril de 2021. A seleção dos artigos foi realizada em abril e maio de 2021 utilizando os descritores COVID-19, cardiopatias, transtornos respiratórios, infarto agudo do miocárdio, trombose venosa profunda, nos idiomas português e inglês. Resultados: 4 artigosfram incluídos nesta pesquisa onde foi identificado que a COVID-19 está associada acomplicações nos sistemas respiratório e cardiovascular. Conclusão: Com o levantamento realizado, verificou-se que existe um maior volume de estudos constatando a vulnerabilidade do sistema cardiovascular a doença, justificado pelas alterações presentes no ECG, aumento da taxa de biomarcadores de IAM e acentuação nos casos de TVP e EP. Verifica-se que novas pesquisas podem ampliar o conhecimento sobre as repercussões cardiorrespiratórias decorrentes da COVID-19.

Palavras-chave: COVID-19; Cardiopatias; Transtornos respiratórios; Infarto agudo do miocárdio; Trombose venosa profunda.

\begin{abstract}
Aim: To disseminate information about the cardiorespiratory repercussions of COVID-19. Methodology: A literature review with bibliographic search in LILACS, SciELO, PubMed and PEDro databases considering clinical trials and observational studies published from July 2020 to April 2021. The selection of articles was made in April and May 2021 using the descriptors COVID-19, heart diseases, respiratory disorders, acute myocardial infarction, deep vein thrombosis, in Portuguese and English. Results: 4 articles were included in this search where it was identified that COVID-19 is associated with complications in the respiratory and cardiovascular systems. Conclusion: With the survey, it was found that there is a greater volume of studies confirming the vulnerability of the cardiovascular system to the disease, justified by the changes present in the ECG, increased rate of biomarkers of AMI and accentuation in cases of DVT and PE. It is verified that further research may expand the knowledge about the cardiorespiratory repercussions resulting from COVID-19.
\end{abstract}

Keywords: COVID-19; Heart diseases; Respiration disorders; Myocardial infarction; Deep vein thrombosis.

\begin{abstract}
Resumen
Objetivo: Difundir información sobre las repercusiones cardiorrespiratorias de COVID-19. Metodología: Revisión de la literatura con levantamiento bibliográfico en las bases de datos LILACS, SciELO, PubMed y PEDro considerando los ensayos clínicos y estudios observacionales publicados en el período de julio de 2020 a abril de 2021. La selección de artículos se realizó en abril y mayo de 2021 utilizando los descriptores COVID-19, cardiopatías, trastornos respiratorios, infarto agudo de miocardio, trombosis venosa profunda, en idiomas portugués e inglés. Resultados: En esta búsqueda se incluyeron 4 artículos en los que se identificó que el COVID-19 está asociado a complicaciones en los sistemas respiratorio y cardiovascular. Conclusión: Con el levantamiento realizado, se verificó que existe un mayor volumen de estudios que constatan la vulnerabilidad del sistema cardiovascular a la enfermedad, justificado por las alteraciones presentes en el ECG, el aumento de la tasa de biomarcadores de IAM y la acentuación en los casos de TVP y EP. Se comprueba que nuevas investigaciones pueden ampliar el conocimiento sobre los efectos cardiorrespiratorios decentes del COVID-19.
\end{abstract}

Palabras clave: COVID-19; Cardiopatías; Trastornos respiratorios; Infarto agudo de miocardio; Trombosis venosa profunda.

\section{Introdução}

O coronavírus (CoV) é o nome originado da família de vírus, descoberta desde a décadade 1960. No ano de 2002, foi descoberta uma variante agressiva desse vírus o SARS-CoV, queocasionou a Síndrome Respiratória Aguda Grave (SARS) e em 2012 o MERS-CoV, SíndromeRespiratória do Oriente Médio que recebeu essa denominação devido a localização dos casos confirmados. Em 2019, na cidade de Wuhan na China, um surto de pneumonia de etiologia não identificada, deixou as autoridades globais em saúde apreensivas em razão de sua alta taxa de transmissibilidade. Após estudos realizados, a patologia foi diferenciada de uma pneumonia, e foi descoberta uma infecção viral por um novo tipo de coronavírus, o SARS-CoV-2 (Abdelghany et al., 2020; Yan et al., 2020; Esakandari et al., 2020; Marson \& Ortega, 2020).

A World Health Organization (OMS) posteriormente denominou essa infecção viralde Corona Virus Disease (COVID19) que traduzindo, seria doença do coronavírus e o numera 119 faz menção ao ano de surgimento da patologia, que recebeu essa nomenclatura com intuito de evitar provocações em grupos religiosos ou étnicos. Em 11 de março de 2020, foi declarada uma pandemia da doença, que mundialmente, até o dia 24 de março de 2021, foram contabilizados 124.215 .843 casos confirmados e 
2.734.374 óbitos. Nacionalmente, 12.220.011 casos confirmados da doença e 300.685 óbitos, enquanto na região Nordeste os números vêm crescendo e atingiram 2.815 .135 casos confirmados e 66.363 óbitos. Chegando à 336.236 casosconfirmados em Pernambuco, sendo 83.917 em Recife e 15.620 casos confirmados em Olinda.(Brasil, 2021;Pernambuco, 2021; Who, 2020; Jin et al., 2020).

A transmissão da COVID-19 ocorre principalmente através de gotículas respiratórias expelidas de uma pessoa contaminada, assim como no contato com superfícies infectadas. Uma vez diagnosticado com a doença, o indivíduo poderá cursar com sintomatologias frequentes como febre, tosse seca, dor de garganta e falta de ar. A doença é manifestada primeiramente no sistema respiratório, mas também apresenta repercussões no sistema digestivo, devido a achados que afirmam que a Enzima Conversora de Angiotensina 2 (ACE2), o principal receptordo SARS-CoV-2, é expressa no trato gastrointestinal (Wiersinga et al., 2020; Mao et al., 2020).

As suas manifestações clínicas costumam variar, podendo não apresentar sintomas (assintomáticos) como sintomas graves resultando em pneumonia, lesões cardíacas e cerebrais. Na maioria das vezes os seus indícios são representados por náuseas, febre, cefaleia, confusões mentais, distúrbios vasculares, hemorragia intracerebral aguda, trombose, encefalopatias, aumento da frequência respiratória e diminuição da saturação de oxigênio (Aguiar et al., 2021; Oliveira et al., 2021)

A COVID-19 possui capacidades neuroinvasivas e pode se distribuir para o Sistema Nervoso Central (SNC) gerando inflamação e desmielinização, podendo causar principalmenteconvulsões, encefalite e Acidente Vascular Encefálico (AVE). Como também pode prejudicar o funcionamento dos rins, ocasionando uma lesão tubular renal. Além disso, cerca de 20 a $35 \%$ dos casos de óbitos apresentavam alguma doença cardiovascular preexistente, e a partir desse achado, foi observada a influência da COVID-19 nesse sistema (Asadi-Pooya \& Simani, 2020; Han \& Ye, 2021; Rocco et al., 2020).

Descobriu-se também que aproximadamente $29 \%$ dos casos que apresentavam manifestações graves da COVID-19, cursavam com lesões cardíacas podendo ser lesão miocárdica, miocardite, infarto agudo do miocárdio (IAM), insuficiência cardíaca, disritmias e eventos tromboembólicos venosos. Onde corroborou uma letalidade geral de 2,3\%, posto que em pacientes com enfermidades cardiovasculares esse índice pode chegar a 10.5\%. A injúria miocárdica decorre em 7\% a 20\% dos indivíduos com COVID-19, podendo potencializar a seriedade da patologia, miocardite ou infarto agudo do miocárdio (IAM) (Rocha et al.,2021; Long et al., 2020; Pegado et al., 2020).

Portanto, se faz necessário encaminhar para a população e os profissionais de saúde, que atuam no enfrentamento à COVID-19, dados acerca das complicações cardiovasculares e respiratórias nesta patologia. Diante disto, este estudo tem o objetivo de realizar um levantamento na literatura cientifica recente, a respeito das repercussões cardiorrespiratórias da COVID19.

\section{Metodologia}

Refere-se a uma revisão integrativa de literatura. O seguinte estudo foi produzido na seguinte ordem: 1) formação da pergunta norteadora; 2) pesquisas e coletas nas bases de dados; 3) observação analítica dos estudos incluídos; 4) discussão dos resultados.

A pergunta norteadora abordada neste estudo foi: "Quais são as repercussões cardiorrespiratórias decorrentes da COVID-19?". Com isso, elaborando um levantamento bibliográfico nasbases de dados Literatura Latino-americana em Ciências da Saúde (LILACS), ScientificElectronic Library Online (SciELO), Public Medline (PubMed) e Physiotherapy Evidence Database (PEDro), sobre o assunto de interesse publicado no período de outubro de 2020 e novembro de 2021.

A triagem dos artigos foi executada entre abril e maio de 2021, utilizando os descritores nos idiomas inglês e português, e o conector booleano AND. Os descritores selecionados para a busca dos artigos foram: COVID-19, cardiopatias, transtornos respiratórios, infarto agudo do miocárdio, trombose venosa profunda. 
Os estudos foram selecionados inicialmente pela leitura de título e resumo. Em sequência foi realizada a leitura de texto completo, para garantia ou não da adequação aos critérios de inclusão. Foi realizada uma síntese de cada estudo, trazendo suas principais informações.

Os critérios de inclusão dos artigos da presente revisão foram: publicações completas em periódicos nacionais e internacionais; artigos que abordassem a temática do estudo, ou seja, as repercussões cardiorrespiratórias consequentes da COVID-19, estudos que contemplem pacientes sem diagnóstico prévio de doença cardiopulmonar. Os critérios de não inclusão envolveram as publicações duplicadas, como também atualizações de estudos já existentes.

A análise dos dados extraídos foi realizada de forma descritiva, em duas etapas. Na primeira, foram identificadas informações quanto à localização do artigo, ano e periódico de publicação, autoria, objetivo, metodologia, resultados principais. Na segunda etapa ocorreu a análise crítica dos artigos, onde foram elencados os avanços evidenciados pelos estudos, quaislacunas teóricas e "práticas" a serem preenchidas e perspectivas a serem consideradas em futuros estudos.

\section{Resultados e Discussão}

Considerando o método que foi exposto, através da busca nas bases de dados mencionadas e análise dos estudos selecionados, foram encontrados esses resultados que se mostram resumidos e esquematizados através da Figura 1.

Figura 1 - Fluxograma da revisão da literatura.

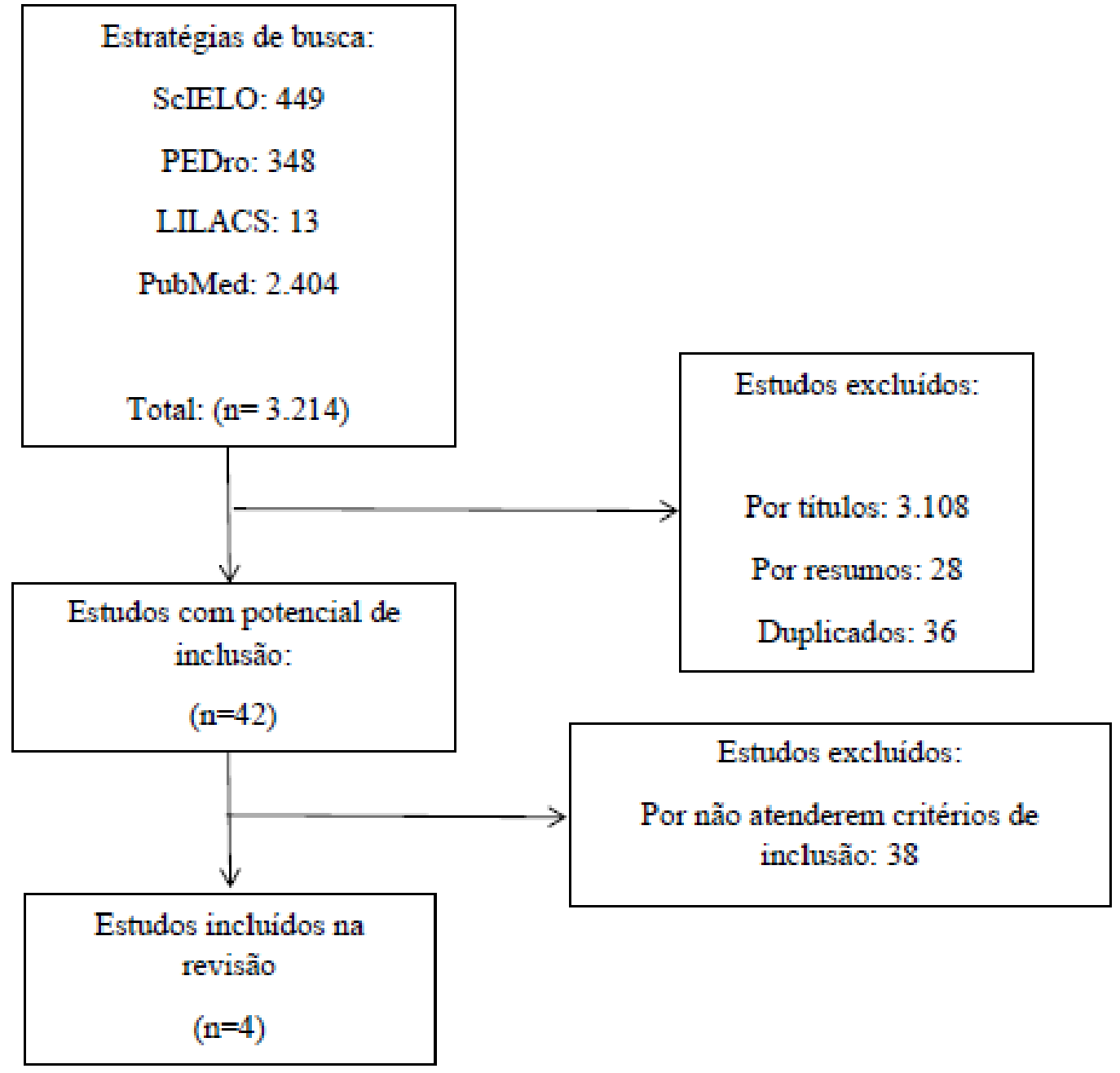

Fonte: Autoria própria. 
Na Tabela 1 foi feita uma relação entre os artigos selecionados para o estudo. Verificou-se que a maior parte das complicações consequentes a COVID-19, que foramobservadas envolve as repercussões cardiovasculares, que variam desde o aumento nas taxas de biomarcadores de IAM, até a associação de casos da doença com uma maior incidência de Trombose Venosa Profunda (TVP), que a sugere como fator de risco para casos de lesão endotelial. Restando um estudo que aborda a embolia pulmonar como repercussão da COVID-19 no sistema respiratório.

Tabela 1 - Dados extraídos dos artigos incluídos na revisão de literatura.

\begin{tabular}{|c|c|c|c|c|}
\hline AUTOR/ANO & AMOSTRA & OBJETIVO & INTERVENÇÃO & RESULTADOS \\
\hline Liaqat et al.,2021 & $\begin{array}{l}201 \text { pacientes com } \\
\text { COVID-19 } \\
\text { no HospitalMilitar do } \\
\text { Paquistão. }\end{array}$ & $\begin{array}{l}\text { Investigar os efeitos da } \\
\text { COVID-19 na lesão } \\
\text { miocárdica em pacientes } \\
\text { hospitalizados quetiveram } \\
\text { teste positivo para } \\
\text { pneumonia COVID-19. }\end{array}$ & $\begin{array}{l}\text { Os padrões de lesão } \\
\text { miocárdica foram } \\
\text { analisados através de } \\
\text { Ecocardiografia e } \\
\text { Ecocardiografia } \\
\text { Transtorácica. }\end{array}$ & $\begin{array}{c}\text { Constatou-se a contribuição } \\
\text { direta da COVID-19 para a } \\
\text { lesão miocárdica nesses } \\
\text { pacientes. A doença leva } \\
\text { diretamente a danos } \\
\text { cardiovasculares em } \\
\text { pacientes críticos e não } \\
\text { críticos. }\end{array}$ \\
\hline Ileri et al.,2021 & $\begin{array}{c}140 \text { pacientes } \\
\text { consecutivos com } \\
\text { pneumonia COVID-19, } \\
\text { com } \\
\text { idade entre } 55 \pm 16 \text { anos, } \\
\text { sendo } \\
82 \text { indivíduos dosexo } \\
\text { masculino. }\end{array}$ & $\begin{array}{c}\text { Explorar a relaçãoentre os } \\
\text { níveis de troponina e a } \\
\text { gravidade da COVID-19. }\end{array}$ & $\begin{array}{c}\text { Tomografia } \\
\text { Computadorizada(TC) de } \\
\text { tórax. }\end{array}$ & $\begin{array}{c}\text { Pacientes com COVID-19 } \\
\text { com } \\
\text { manifestações graves de } \\
\text { TC, progressão da doença e } \\
\text { necessidade de UTI têm } \\
\text { níveis detroponina } \\
\text { significativamentemais } \\
\text { elevados. }\end{array}$ \\
\hline Zhang et al.,2021 & $\begin{array}{c}143 \text { pacientes com } \\
\text { COVID-19 } \\
\text { hospitalizados de } 29 \text { de } \\
\text { janeiro de } 2020 \text { a } 29 \text { de } \\
\text { fevereiro de } 2020 \text { com } \\
\text { idade } \\
\text { entre } 63 \pm 14 \\
\text { anos, sendo } 74 \text { do sexo } \\
\text { masculino. }\end{array}$ & $\begin{array}{c}\text { Investigar a Trombose } \\
\text { Venosa Profunda (TVP) em } \\
\text { pacientes hospitalizados com } \\
\text { COVID-19. }\end{array}$ & $\begin{array}{c}\text { Análise dos dados } \\
\text { demográficos, clínicos e } \\
\text { laboratoriais, incluindo } \\
\text { ultrassonografia dos } \\
\text { membros inferiores e } \\
\text { variáveis de desfecho foram } \\
\text { obtidos e comparados entre } \\
\text { grupos com e sem } \\
\text { TVP. }\end{array}$ & $\begin{array}{l}\text { O estudo sugere que a } \\
\text { COVID-19 é } \\
\text { provavelmente um fator de } \\
\text { risco adicional para TVP } \\
\text { em pacienteshospitalizados. }\end{array}$ \\
\hline Kwok et al.,2021 & $\begin{array}{l}\text { Pacientes adultos com } \\
\text { Embolia Pulmonar (EP) } \\
\text { aguda que receberam } \\
\text { atendimento em um } \\
\text { sistema hospitalar } \\
\text { acadêmico na cidade de } \\
\text { Nova York entre } 1^{\circ} \text { de } \\
\text { março e } 30 \text { de } \\
\text { abril de } 2020 .\end{array}$ & $\begin{array}{c}\text { Investigar se a utilização, } \\
\text { recomendações e resultados } \\
\text { das Equipes de Resposta à } \\
\text { Embolia Pulmonar(EREP) } \\
\text { para pacientes com } \\
\text { diagnóstico de EP aguda } \\
\text { mudaram durante a } \\
\text { pandemia da } \\
\text { COVID-19. }\end{array}$ & ıálise de coorteretrospectiva. & $\begin{array}{l}\text { As recomendações e } \\
\text { resultados das EREPs } \\
\text { foram semelhantes antes e } \\
\text { durante a pandemia, e } \\
\text { levaram à diminuição do } \\
\text { tempo de internação. }\end{array}$ \\
\hline
\end{tabular}

Fonte: Autoria própria.

De acordo com Kwok et al., (2020) a COVID-19 afeta o sistema respiratório e cardiovascular e uma das complicações decorrentes da doença é a EP. e Liaqat et al., (2021); Ileri et al., (2021); Zhang et al., (2020) confirmam essa afirmação devido ao 
aumento na taxa de biomarcadores de infarto e ocorrência de eventos tromboembólicos venosos nos pacientes hospitalizados com esta doença.

Através do estudo Liaqat et al., (2021) constata a sua gravidade e alterações no ECG, devido a ACE2 que é receptor de ligação para o SARS-CoV-2, sendo altamente expressa nos pericitos cardíacos, indicando a susceptibilidade do sistema cardiovascular diante da doença. As altas taxas de troponina cardíaca (TnC) que é um importante biomarcador para o IAM em casos de COVID-19, vem sendo observadas desde os primeiros estudos publicados e esses dados sugerem a contribuição da patologia para o aumento de casos graves da doença e o seu crescente número de óbitos.

Na pesquisa realizada, os 201 pacientes do Hospital Militar do Paquistão foram divididos em 2 grupos, sendo eles críticos quando se fazia necessário cuidados intensivos e o suporte ventilatório através da Ventilação Mecânica Invasiva (VMI), e não críticos quando a VMI era dispensável. Foi constatado que 54\% da amostra aumentaram os níveis de troponinaligada a actina (TNI) sugerindo lesão miocárdica, e 47,2\% dos pacientes pertencem ao grupo crítico. Embora os valores médios tenham sido elevados em ambos os grupos, os níveis de TN foram significativamente mais elevados $>0,2 \mathrm{ng} / \mathrm{ml}$ em $\mathrm{pacientes} \mathrm{críticos}$ (Liaqat et al., 2021).

O segundo estudo demonstrado (Tabela1), corrobora com a afirmação que níveis altos de TnC estão ligados a vulnerabilidade do sistema cardiovascular à COVID-19. Através da análise dos achados tomográficos do tórax de 140 pacientes admitidos em uma unidade hospitalar da Turquia, que 87,9\% apresentaram infiltrações pulmonares bilaterais e 47,1\% resultados moderados e graves de TC de tórax. Foi observado que cerca de 8 à $28 \%$ dos indivíduos que tinham manifestações graves da COVID-19, progressão da doença no acometimento pulmonar e necessidade de VMI, mostraram um nível elevado de TnC sugerindolesão do miocárdio e indicando o uso dos níveis de TnC na estratificação de risco destes pacientes (Ileri et al., 2021).

Continuando com a investigação da relação da COVID-19 com o comprometimento dosistema cardiovascular, Zhang et al., (2020) relata em seu estudo que foi realizado com 143 pacientes com COVID-19 admitidos no West Branch of Union Hospital em Wuham na China,através de achados clínicos e da ultrassonografia venosa em membros inferiores, constatou-se a prevalência de TVP em 46,1\% da amostra, sendo maior que em outros pacientes hospitalizados graves, críticos ou de alto risco. Logo, a COVID-19 é um suposto fator de riscocomplementar para TVP em pacientes hospitalizados.

Em seu estudo Kwok et al., (2020) relatou que a COVID-19 está correlacionada ao aumento no número de casos de TVP e EP, em virtude da hipercoagulabilidade ocasionada pela doença. Através da análise dos prontuários de 82 pacientes adultos do Sistema Hospitalar Acadêmico da cidade de Nova York, houve a investigação de variáveis como sexo, idade e presença de comorbidades. Verificou-se que 44 pacientes eram COVID-positivos com ativação das EREPs em $16 \%$ dos casos. As EREPs são formadas por médicos de diversas especialidades que atuam na avaliação e tratamento de forma rápida dos pacientes com EP.

Quando são comparados dados referentes a ativação das EREPs antes e durante a pandemia da COVID-19, percebe-se uma redução nos números de chamadas, que segundo Kwok et al., (2020) pode estar envolvido com um maior preparo da equipe intensivista e com isso, não se fez necessária informações extras, por outro lado a base fornecida pelo estudo reforçam que houve uma maior incidência de casos de EP em pacientes com COVID-19. De todo modo parece possível constatar que a COVID-19 está associada a complicaçõesnos sistemas respiratório e cardiovascular. Liaqat et al., (2021); Ileri et al., (2021); Zhang et al., (2020); Kwok et al., (2020), no entanto, verifica-se que novas pesquisas podem ampliar oconhecimento sobre as repercussões cardiorrespiratórias decorrentes da COVID-19.

\section{Conclusão}

Diante do levantamento que foi realizado, verificou-se que apesar da COVID-19 afetar primariamente o sistema respiratório, existe um maior volume de estudos confirmando a vulnerabilidade do sistema cardiovascular a doença. Isso é justificado pelas alterações identificadas no ECG dos pacientes em estudo e a elevação da taxa de biomarcadores para IAMque 
são descritas desde as primeiras pesquisas elaboradas pontuando a susceptibilidade do sistema cardiovascular. Constatou-se também o aumento dos casos de TVP em pacientes hospitalizados, sugerindo qua a doença como um suposto fator de risco complementar para TVP. Além da acentuação do número de manifestações de EP nesses pacientes, devido a hipercoagulabilidade ocasionada pela COVID-19.

Em razão da pandemia ainda estar em curso e o curto período de recorte do tempo para realização da busca nas bases de dados, considerando as complicações não preexistentes da COVID-19 no sistema cardiorrespiratório, o número de artigos encontrados foi reduzido, logo se faz necessária uma abordagem mais aprofundada para uma maior compreensão do tema.

\section{Referências}

Abdelghany, T. M., Ganash, M., Bakri, M. M., Elhussieny, N. I., Qanash, H., \& Al-Rajhi, A. M. H. (2020). A review SARS-CoV-2 the other face to SARS$\mathrm{CoV}$ and MERS-CoV: About future predictions. Biomedical Journal. https://doi.org/10.1016/j.bj.2020.10.008

Aguiar, B. F., Sarquis, L. M. M., \& Miranda, F. M. D. (2021). Sequelae of Covid-19: a reflection on the impacts on the health of the worker. Research, Society and Development, 10(14), e40101421886. https://doi.org/10.33448/rsd-v10i14.21886

Asadi-Pooya, A. A., \& Simani, L. (2020). Central nervous system manifestations of COVID-19: A systematic review. Journal of the Neurological Sciences, 413, 116832. https://doi.org/10.1016/j.jns.2020.116832

Brasil. (2020). Ministério da Saúde. Secretaria de Vigilância em Saúde. Painel Coronavírus. https://www.coronavirus.ms.gov.br/?cat=5

Esakandari, H., Nabi-Afjadi, M., Fakkari-Afjadi, J., Farahmandian, N., Miresmaeili, S.- M., \& Bahreini, E. (2020). A comprehensive review of COVID-19 characteristics. Biological Procedures Online, 22(1). https://doi.org/10.1186/s12575-020-00128-2

Han, X., \& Ye, Q. (2020). Kidney involvement in COVID-19 and its treatments. Journal of Medical Virology. https://doi.org/10.1002/jmv.26653

Ileri, C., Dogan, Z., Ozben, B., Karaoglu, C., Gunay, N., Tigen, K., Basat, S., \& Uyan, C. (2021). Evaluation of the relation between cardiac biomarkers and thorax computed tomography findings in COVID-19 patients. Biomarkers in Medicine, 15(4), 285-293. https://doi.org/10.2217/bmm-2020-0388

Jin, Y., Yang, H., Ji, W., Wu, W., Chen, S., Zhang, W., \& Duan, G. (2020). Virology, Epidemiology, Pathogenesis, and Control

Kwok, B., Brosnahan, S. B., Amoroso, N. E., Goldenberg, R. M., Heyman, B., Horowitz, J. M., Jamin, C., Sista, A. K., Smith, D. E., Yuriditsky, E., \& Maldonado, T. S. (2020). Pulmonary Embolism Response Team activation during the COVID-19 pandemic in a New York City Academic Hospital: a retrospective cohort analysis. Journal of Thrombosis and Thrombolysis. https://doi.org/10.1007/s11239-020-02264-8

Liaqat, A., Ali-Khan, R. S., Asad, M., \& Rafique, Z. (2021). Evaluation of myocardial injury patterns and ST changes among critical and non-critical patients with coronavirus-19 disease. Scientific Reports, 11(1). https://doi.org/10.1038/s41598-021-84467-4

Long, B., Brady, W. J., Koyfman, A., \& Gottlieb, M. (2020). Cardiovascular complications in COVID-19. The American Journal of Emergency Medicine, 38(7), 1504-1507. https://doi.org/10.1016/j.ajem.2020.04.048

Mao, R., Qiu, Y., He, J.-S., Tan, J.-Y., Li, X.-H., Liang, J., Shen, J., Zhu, L.-R., Chen, Y., Iacucci, M., Ng, S. C., Ghosh, S., \& Chen, M.-H. (2020). Manifestations and prognosis of gastrointestinal and liver involvement in patients with COVID-19: a systematic review and meta-analysis. The Lancet Gastroenterology \& Hepatology, 5(7), 667-678. https://doi.org/10.1016/s2468-1253(20)30126-6

Marson, F. A. L., \& Ortega, M. M. (2020). COVID-19 in Brazil. Pulmonology, 26(4), 241-244. https://doi.org/10.1016/j.pulmoe.2020.04.008 of COVID-19. Viruses, 12(4), 372. https://doi.org/10.3390/v12040372

Oliveira, B. C. de ., Santos , F. C. dos, Silva, H. G. N., Castro, I. O., Franco, V. de S. P. ., Silva, C. de S. e ., Souza, S. M. de O., Silva, V. M. B. da ., França, L. G. L. ., \& Ferreira, L. G. de F. (2021). Epidemiological and clinical profile of patients with Covid-19 in an Intensive Care Unit of a public hospital in TeresinaPI. Research, Society and Development, 10(14), e563101422053. https://doi.org/10.33448/rsd-v10i14.22053

Pegado, R., Silva-Filho, E., Lima, I. N. D. F., \& Gualdi, L. (2020). Coronavirus disease 2019 (COVID-19) in Brasil: information to physical therapists. Revista da Associação Médica Brasileira, 66(4), 498-501. https://doi.org/10.1590/1806-9282.66.4.498

Pernambuco. (2021). Secretaria Estadual de Saúde de Pernambuco. Boletim COVID-19 SES -PE. https://www.pecontracoronavirus.pe.gov.br

Rocco, I. S., Gomes, W. J., Viceconte, M., Bolzan, D. W., Moreira, R. S. L., Arena, R., \& Guizilini, S. (2020). Cardiovascular involvement in COVID-19: not to be missed. Brazilian Journal of Cardiovascular Surgery, 35(4), 530-538. https://doi.org/10.21470/1678-9741-2020-0224

Rocha, N. P. M. ., Santos, N. V. dos ., Souza, R. A. ., Silva, I. de A. C. e ., Oliveira, A. M. de O. . and Moraes, D. D. P. de . (2021) "Cardiovascular manifestation in COVID-19 infection in a patient with rheumatoid arthritis: case report ", Research, Society and Development, 10(14), p. e456101422277. doi: 10.33448/rsdv10i14.22277.

Wiersinga, W. J., Rhodes, A., Cheng, A. C., Peacock, S. J., \& Prescott, H. C. (2020). Pathophysiology, Transmission, Diagnosis, and Treatment of Coronavirus Disease 2019 (COVID-19). JAMA, 324(8), 782-793. https://doi.org/10.1001/jama.2020.12839

World Health Organization. (2020). announces name for disease causedby new coronavirus: COVID-19. http://encurtador.com.br/erOV4 
Research, Society and Development, v. 11, n. 2, e43011225746, 2022

(CC BY 4.0) | ISSN 2525-3409 | DOI: http://dx.doi.org/10.33448/rsd-v11i2.25746

World Health Organization. (2021). Coronavirus Disease (COVID-19) Dashboard. Disponível em: https://covid19.who.int

Yan, Y., Chang, L., \& Wang, L. (2020). Laboratory testing of SARS-CoV, MERS-CoV, and SARS-CoV-2 (2019-nCoV): Current status, challenges, and countermeasures. Reviews in Medical Virology, 30(3). https://doi.org/10.1002/rmv.2106

Zhang, L., Feng, X., Zhang, D., Jiang, C., Mei, H., Wang, J., Zhang, C., Li, H., Xia, X., Kong, S., Liao, J., Jia, H., Pang, X., Song, Y., Tian, Y., Wang, B., Wu, C., Yuan, H., Zhang, Y., Xie, M. (2020). Deep Vein Thrombosis in Hospitalized Patients With COVID-19 in Wuhan, China. Circulation, 142(2), 114128. https://doi.org/10.1161/circulationaha.120.046702 